\title{
Brief Psychotic Disorder
}

National Cancer Institute

\section{Source}

National Cancer Institute. Brief Psychotic Disorder. NCI Thesaurus. Code C94390.

A disorder characterized by delusions, hallucinations, disorg anized speech, and/or grossly disorganized behavior that resolve within a month. 